\title{
Joint 2D-DOA and Frequency Estimation for L-Shaped Array Using Iterative Least Squares Method
}

\author{
Ling-yun Xu, ${ }^{1,2}$ Xiao-fei Zhang, ${ }^{1}$ Zong-ze $\mathrm{Xu},{ }^{1}$ and Miao $\mathrm{Yu}^{2}$ \\ ${ }^{1}$ College of Electronic Information Engineering, Nanjing University of Aeronautics and Astronautics, Nanjing 210016, China \\ ${ }^{2}$ Nanjing Telecommunication Technology Institute, Nanjing 210007, China
}

Correspondence should be addressed to Ling-yun Xu, xlyun@nuaa.edu.cn

Received 19 May 2012; Revised 20 July 2012; Accepted 24 July 2012

Academic Editor: Jung-Chieh Chen

Copyright ( $) 2012$ Ling-yun Xu et al. This is an open access article distributed under the Creative Commons Attribution License, which permits unrestricted use, distribution, and reproduction in any medium, provided the original work is properly cited.

\begin{abstract}
We introduce an iterative least squares method (ILS) for estimating the 2D-DOA and frequency based on L-shaped array. The ILS iteratively finds direction matrix and delay matrix, then 2D-DOA and frequency can be obtained by the least squares method. Without spectral peak searching and pairing, this algorithm works well and pairs the parameters automatically. Moreover, our algorithm has better performance than conventional ESPRIT algorithm and propagator method. The useful behavior of the proposed algorithm is verified by simulations.
\end{abstract}

\section{Introduction}

Antenna arrays have been used in many fields such as radar, sonar, and mobile communications, and so forth, [1-6]. The direction of arrival and frequency estimation of signals impinging on an array of sensors have received considerable attention in the field of array signal processing. For example, these parameters can be applied to locate the mobiles and allocate pilot tones in space division multiple access (SDMA) systems. Furthermore, a precise estimation of these parameters is helpful to attain a better channel estimate and thus enhances the system performance [7]. Uniform linear arrays for estimation of wave arrival have been studied extensively, and they contain maximum likelihood (ML) [8], multiple signal classification (MUSIC) algorithm $[9,10]$, estimation of signal parameters via rotational invariance techniques (ESPRIT) $[11,12]$, propagator method (PM) [13], and so forth.

The ML method is often applicable but might be computationally prohibitive. ESPRIT and MUSIC algorithms are based on signal subspace and have better parameter estimation performance. The main advantages of MUSIC/ESPRIT are the high-resolution estimates of direction of arrivals (DOAs) and frequencies, while the computational effort compared to ML method is significantly reduced. MUSIC requires multiple dimensional spectral peak searching, and it is the search that is still computationally expensive. The primary computational advantage of ESPRIT is that it eliminates the search procedure inherent. ESPRIT method requires eigenvalue decomposition (EVD) to the cross spectral matrix or singular value decomposition (SVD) to the received data. Reference [13] presented propagator method to estimate the angle and frequency with uniform linear array. Propagator method has low complexity, but its parameters' estimation performance is less than ESPRIT algorithm. Compared with uniform linear array, L-shaped array can identify $2 \mathrm{D}-\mathrm{DOA}$ and is very close to actual situation [14, 15]. And thus we propose a novel iterative-based angle and frequency estimation algorithm with L-shaped array which can achieve better performance than ESPRIT [11] and propagator method [13]. The proposed algorithm can obtain automatically paired 2D-DOA and frequency estimation. This method is an iterative algorithm, which does not need EVD or SVD, and only requires fewer iterations for convergence. The useful behavior of the proposed algorithm is verified by simulations.

The remainder of this paper is structured as follows. Section 2 develops data model. Section 3 deals with algorithmic issues. Section 4 presents simulation results, and Section 5 summarizes our conclusions. 
Denote. We denote by $(\cdot)^{*}$ the complex conjugation, by $(\cdot)^{T}$ the matrix transpose, and by $(\cdot)^{H}$ the matrix conjugate transpose. $\operatorname{diag}\{\cdot\}$ is to construct a diagonal matrix.

\section{Data Model}

We consider an L-shaped array with sensors at $2 M-1$ different locations as shown in Figure 1. A uniform linear array containing $M$ elements is located in $Y$-axis, and the other uniform linear array containing $M$ elements is located in $X$-axis. We suppose that there are $K$ narrowband uncorrelated signal sources impinge on the L-shaped array with $\left(\phi_{k}, \theta_{k}\right), k=1,2, \ldots, K$, where $\phi_{k}, \theta_{k}$ are the elevation angle and the azimuth angle of the $k$ th source, respectively.

The received signal of $M$ elements in $X$-axis is shown

$$
\mathbf{x}_{1}(t)=\mathbf{A}_{x} \mathbf{s}(t)+\mathbf{w}_{1}(t),
$$

where $\mathbf{s}(t)=\left[s_{1}(t), s_{2}(t), \ldots, s_{K}(t)\right]^{T}$ is the source matrix, $\mathbf{w}_{1}(t)$ is the received additive white Gaussian noise, and $\mathbf{A}_{x}=$ $\left[\mathbf{a}_{x}\left(\theta_{1}, \phi_{1}, f_{1}\right), \mathbf{a}_{x}\left(\theta_{2}, \phi_{2}, f_{2}\right), \ldots, \mathbf{a}_{x}\left(\theta_{K}, \phi_{K}, f_{K}\right)\right] \in \mathbb{C}^{M \times K}$ is the $X$-axis direction matrix, and $\mathbf{a}_{x}\left(\theta_{k}, \phi_{k}, f_{k}\right)=\left[1, u\left(\theta_{k}\right.\right.$, $\left.\left.\phi_{k}, f_{k}\right), \ldots, u^{M}\left(\theta_{k}, \phi_{k}, f_{k}\right)\right]^{T}, u\left(\theta_{k}, \phi_{k}, f_{k}\right)=\exp \left[-j 2 \pi d \cos \theta_{k}\right.$ $\left.\sin \phi_{k} f_{k} / c\right]$.

The received signal of $M$ elements in $Y$-axis is denoted as

$$
\mathbf{x}_{2}(t)=\mathbf{A}_{y} \mathbf{s}(t)+\mathbf{w}_{2}(t),
$$

where $\mathbf{A}_{y}=\left[\mathbf{a}_{y}\left(\theta_{1}, \phi_{1}, f_{1}\right), \mathbf{a}_{y}\left(\theta_{2}, \phi_{2}, f_{2}\right), \ldots, \mathbf{a}_{y}\left(\theta_{K}, \phi_{K}, f_{K}\right)\right] \in$ $\mathbb{C}^{(M-1) \times K}$ is the $Y$-axis direction matrix and $\mathbf{a}_{y}\left(\theta_{k}, \phi_{k}\right.$, $\left.f_{k}\right)=\left[v\left(\theta_{k}, \phi_{k}, f_{k}\right), v^{2}\left(\theta_{k}, \phi_{k}, f_{k}\right), \ldots, v^{M-1}\left(\theta_{k}, \phi_{k}, f_{k}\right)\right]^{T}, v\left(\theta_{k}\right.$, $\left.\phi_{k}, f_{k}\right)=\exp \left[-j 2 \pi d \sin \theta_{k} \sin \phi_{k} f_{k} / c\right], \mathbf{w}_{2}(t)$ is the received white Gaussian noise.

The received signal of the L-shaped array antennas can be denoted as

$$
\mathbf{x}(t)=\left[\begin{array}{l}
\mathbf{x}_{1} \\
\mathbf{x}_{2}
\end{array}\right]=\left[\begin{array}{l}
\mathbf{A}_{x} \\
\mathbf{A}_{y}
\end{array}\right] \mathbf{s}(t)+\mathbf{w}(t)=\mathbf{A s}(t)+\mathbf{w}(t),
$$

where the direction matrix $\mathbf{A}$ and noise matrix $\mathbf{w}(t)$ are shown as follows:

$$
\mathbf{A}=\left[\begin{array}{l}
\mathbf{A}_{x} \\
\mathbf{A}_{y}
\end{array}\right], \quad \mathbf{w}(t)=\left[\begin{array}{l}
\mathbf{w}_{1}(t) \\
\mathbf{w}_{2}(t)
\end{array}\right] .
$$

$\mathbf{A}_{x}$ and $\mathbf{A}_{y}$ are Vandermonde matrices. The delayed signal for (3) with $\tau_{p}$ can be denoted as

$$
\begin{aligned}
\mathbf{x}\left(t-\tau_{p}\right) & =\mathbf{A s}\left(t-\tau_{p}\right)+\mathbf{w}\left(t-\tau_{p}\right) \\
& =\mathbf{A} \Phi_{p} \mathbf{s}(t)+\mathbf{w}\left(t-\tau_{p}\right),
\end{aligned}
$$

where $\boldsymbol{\Phi}_{p}=\operatorname{diag}\left[e^{-j 2 \pi f_{1} \tau_{p}}, e^{-j 2 \pi f_{2} \tau_{p}}, \ldots, e^{-j 2 \pi f_{K} \tau_{p}}\right]$.

We assume that channel state information is constant for $N$ symbols. The received signal of array antennas can be denoted as

$$
\mathbf{X}_{p}=\mathbf{A} \Phi_{p} \mathbf{S}+\mathbf{W}_{p}
$$

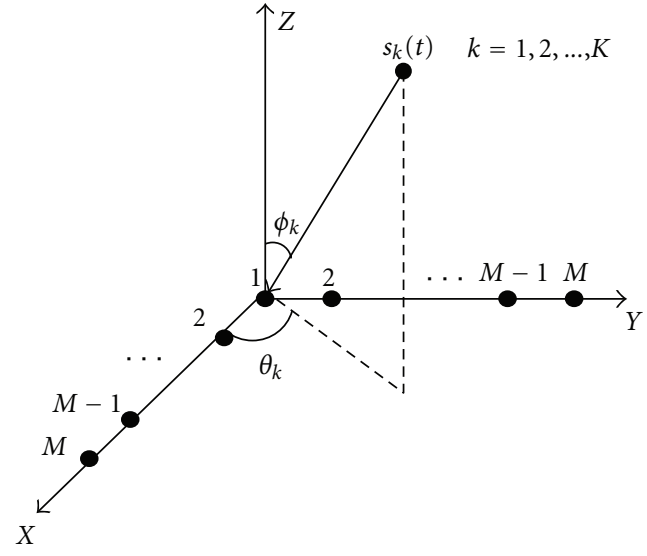

Figure 1: The structure of L-shaped array.

where $\mathbf{W}_{p}=\left[\mathbf{w}\left(t_{1}-\tau_{p}\right), \mathbf{w}\left(t_{2}-\tau_{p}\right), \ldots, \mathbf{w}\left(t_{N}-\tau_{p}\right)\right], \mathbf{S}=$ $\left[\mathbf{s}\left(t_{1}\right), \mathbf{s}\left(t_{2}\right), \ldots, \mathbf{s}\left(t_{N}\right)\right]$.

Define the delay matrix as

$$
\boldsymbol{\Phi}=\left[\begin{array}{cccc}
1 & 1 & \cdots & 1 \\
e^{-j 2 \pi f_{1} \tau_{1}} & e^{-j 2 \pi f_{2} \tau_{1}} & \cdots & e^{-j 2 \pi f_{K} \tau_{1}} \\
\vdots & \vdots & \ddots & \vdots \\
e^{-j 2 \pi f_{1} \tau_{P}} & e^{-j 2 \pi f_{2} \tau_{P}} & \cdots & e^{-j 2 \pi f_{K} \tau_{P}}
\end{array}\right] \in \mathbb{C}^{(P+1) \times K}
$$

Equation (6) is also denoted as

$$
\mathbf{X}_{p}=\mathbf{A} D_{p}(\boldsymbol{\Phi}) \mathbf{S}+\mathbf{W}_{p}, \quad p=1,2, \ldots, P+1,
$$

where $D_{m}(\cdot)$ is to extract the $m$ th row of its matrix argument and constructs a diagonal matrix out of it.

\section{Iterative Least Squares Method}

It can be seen from (8) that if the direction matrices $\mathbf{A}$ and $\boldsymbol{\Phi}$ are obtained, then the estimates of 2D-DOA and frequency follow immediately based on L-shaped array geometries. Hence our main focus in this section is on estimating $\mathbf{A}$ and $\Phi$.

3.1. ILS Cost Function. The cost function can be constructed via the least squares criterion and given by

$$
f\left(\mathbf{A}, D_{1}(\boldsymbol{\Phi}), \ldots, D_{P+1}(\boldsymbol{\Phi}), \mathbf{S}\right)=\sum_{p=1}^{P+1}\left\|\mathbf{X}_{p}-\mathbf{A} D_{p}(\boldsymbol{\Phi}) \mathbf{S}\right\|_{F}^{2}
$$

where \|\|$_{F}^{2}$ denotes the Frobenius norm. Interestingly, if any two subsets of $\mathbf{A},\left\{D_{p}(\boldsymbol{\Phi})\right\}_{p=1}^{P+1}$ and $\mathbf{S}$ are fixed, the remaining parameter subset can be easily obtained by minimizing (9). 
Firstly, fix $\mathbf{A}$ and $\mathbf{S}$. Recall that $\mathbf{A}=\left[\mathbf{a}_{1}, \mathbf{a}_{2}, \ldots, \mathbf{a}_{K}\right], \mathbf{S}=$ $\left[\mathbf{s}_{1}, \mathbf{s}_{2}, \ldots, \mathbf{s}_{K}\right]^{T}$, and $D_{p}(\boldsymbol{\Phi})=\operatorname{diag}\left\{\beta_{1}(p), \beta_{2}(p), \ldots \beta_{K}(p)\right\}$, where $\beta_{k}(p)=e^{-j 2 \pi f_{k} \tau_{p-1}}, k=1,2, \ldots, K$. Then (9) can be rewritten as

$$
\begin{aligned}
f & =\sum_{p=1}^{P+1}\left\|\mathbf{X}_{p}-\sum_{k=1}^{K} \mathbf{a}_{k} \beta_{k}(p) \mathbf{s}_{k}\right\|_{F}^{2} \\
& =\sum_{p=1}^{P+1} \operatorname{tr}\left\{\left[\mathbf{X}_{p}-\sum_{k=1}^{K} \mathbf{a}_{k} \beta_{k}(p) \mathbf{s}_{k}\right]\left[\mathbf{X}_{p}^{H}-\sum_{k=1}^{K} \mathbf{s}_{k}^{H} \beta_{k}^{*}(p) \mathbf{a}_{k}^{H}\right]\right\} .
\end{aligned}
$$

Let the gradient of $f$ with respect to $\beta_{k}^{*}(p)$, be equal to zero, one obtains

$$
\mathbf{a}_{k}^{H}\left[\sum_{k=1}^{K} \mathbf{a}_{k} \beta_{k}(p) \mathbf{s}_{k}\right] \mathbf{s}_{k}^{H}-\mathbf{a}_{k}^{H} \mathbf{X}_{p} \mathbf{s}_{k}^{H}=0,
$$

for $p=1,2, \ldots, P+1$ and $k=1,2, \ldots, K$. The above equation yields

$$
\begin{array}{r}
\mathbf{a}_{k}^{H} \mathbf{A} D_{p}(\Phi) \mathbf{S} \mathbf{s}_{k}^{H}=\mathbf{a}_{k}^{H} \mathbf{X}_{p} \mathbf{s}_{k}^{H}, \quad p=1,2, \ldots, P+1, \\
k=1,2, \ldots, K .
\end{array}
$$

Let $\boldsymbol{\beta}(p)=\left[\beta_{1}(p), \beta_{2}(p), \ldots \beta_{K}(p)\right]^{T}, \mathbf{E}=\left(\mathbf{A}^{H} \mathbf{A}\right) \oplus\left(\mathbf{S S}^{H}\right)^{*}$, and $\mathbf{d}(p)=\left[\mathbf{a}_{1}^{H} \mathbf{X}_{p} \mathbf{s}_{1}^{H}, \ldots, \mathbf{a}_{K}^{H} \mathbf{X}_{p} \mathbf{s}_{K}^{H}\right]^{T}$, where $\oplus$ denotes the Hadamard product. Then (12) can be rewritten in matrix form

$$
\mathbf{E} \boldsymbol{\beta}(p)=\mathbf{d}(p), \quad p=1,2, \ldots, P+1 .
$$

Hence, the estimate of $D_{p}(\boldsymbol{\Phi})$ can be easily computed as

$$
\begin{array}{r}
D_{p}(\boldsymbol{\Phi})=\operatorname{diag}\{\boldsymbol{\beta}(p)\}=\operatorname{diag}\left\{\mathbf{E}^{-1} \mathbf{d}(p)\right\}, \\
p=1,2, \ldots, P+1 .
\end{array}
$$

Secondly, let A and $\left\{D_{p}(\boldsymbol{\Phi})\right\}_{p=1}^{P+1}$ be fixed. Differentiation of (9) with respect to $\boldsymbol{S}$ yields the estimate of $\widehat{\boldsymbol{S}}$

$$
\hat{\mathbf{S}}^{H}=\left[\sum_{p=1}^{P+1} \mathbf{X}_{p}^{H} \mathbf{A} D_{p}^{H}(\boldsymbol{\Phi})\right]\left[\sum_{p=1}^{P+1} D_{p}(\boldsymbol{\Phi}) \mathbf{A}^{H} \mathbf{A} D_{p}^{H}(\boldsymbol{\Phi})\right]^{-1} .
$$

Thirdly fix $\left\{D_{p}(\boldsymbol{\Phi})\right\}_{p=1}^{P+1}$, and S. Let the gradient of (9) with respect to $\mathbf{A}$ be equal to zero, we have

$$
\sum_{p=1}^{P+1}\left[\mathbf{A} D_{p}(\boldsymbol{\Phi}) \mathbf{S}-\mathbf{X}_{p}\right] \mathbf{S}^{H} D_{p}^{H}(\boldsymbol{\Phi})=0,
$$

which gives the estimate of $\mathbf{A}$

$$
\widehat{\mathbf{A}}=\left[\sum_{p=1}^{P+1} \mathbf{X}_{p} \mathbf{S}^{H} D_{p}^{H}(\boldsymbol{\Phi})\right]\left[\sum_{p=1}^{P+1} D_{p}(\Phi) \mathbf{S} \mathbf{S}^{H} D_{p}^{H}(\boldsymbol{\Phi})\right]^{-1} .
$$

Based on the above analysis, the ILS algorithm is summarized as follows.

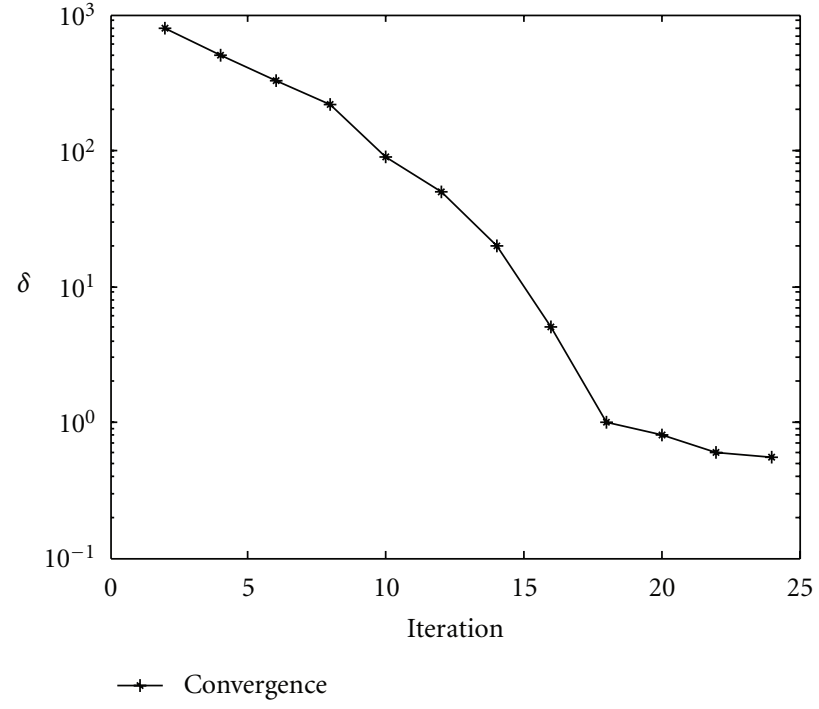

FIgURE 2: Performance of algorithmic convergence.

Let the initial values $\hat{\mathbf{A}}_{0}=\overline{\mathbf{A}} \in \mathbb{C}^{(2 M-1) \times K}$ and $\widehat{\mathbf{S}}_{0}=\overline{\mathbf{S}} \in$ $\mathbb{C}^{K \times N}$, the elements of $\overline{\mathbf{A}}$ and $\overline{\mathbf{S}}$ are equal to 1 . For iteration index $i=1,2, \ldots$, do the following three steps.

Step 1. Calculate $\left\{D_{p}(\boldsymbol{\Phi})_{i}\right\}$ in (14) using $\widehat{\mathbf{A}}_{i-1}$ and $\widehat{\boldsymbol{S}}_{i-1}$, for $p=1,2, \ldots, P+1$.

Step 2. Calculate $\widehat{\mathbf{S}}_{i}$ in (15) using $\left\{D_{p}(\boldsymbol{\Phi})_{i}\right\}_{p=1}^{P+1}$ and $\hat{\mathbf{A}}_{i-1}$.

Step 3. Calculate $\hat{\mathbf{A}}_{i}$ in (17) using $\hat{\boldsymbol{S}}_{i}$ and $\left\{D_{p}(\boldsymbol{\Phi})_{i}\right\}_{p=1}^{P+1}$.

$\delta=\left\|\widehat{\mathbf{A}}_{i}-\widehat{\mathbf{A}}_{i-1}\right\|_{F}^{2}$ with a threshold $\xi(0<\xi \leq 1)$; if $\delta_{i}<\xi$, then stop; otherwise, go to Step 1.

The basic idea of the iterative least square algorithm is to minimize the cost function $f\left(\mathbf{A}, D_{1}(\boldsymbol{\Phi}), \ldots, D_{P+1}(\boldsymbol{\Phi}), \mathbf{S}\right)$ with respect to $\mathbf{A},\left\{D_{p}(\boldsymbol{\Phi})\right\}_{p=1}^{P+1}$, and $\mathbf{S}$ by using the cyclic minimization (CM) technique [16], which monotonically decreases the cost function. Furthermore, we know $\sum_{p=1}^{P+1}\left\|\mathbf{X}_{p}-\mathbf{A} D_{p}(\boldsymbol{\Phi}) \mathbf{S}\right\|_{F}^{2} \geq 0$. Hence, the proposed algorithm is convergent, only 20 iterations are required to achieve convergence for this iterative algorithm (also shown in Figure 2).

We obtain the estimated matrices $\hat{\mathrm{A}}=\mathbf{A} \Pi \boldsymbol{\Delta}_{1}, \widehat{\boldsymbol{\Phi}}=$ $\boldsymbol{\Phi} \Pi \Delta_{2}$, and $\hat{\mathbf{S}}=\mathbf{S} \Pi \Delta_{3}$, where $\Pi$ is a permutation matrix and $\Delta_{1}, \Delta_{2}, \Delta_{3}$ stand for diagonal scaling matrices satisfying $\Delta_{1} \Delta_{2} \Delta_{3}=\mathbf{I}_{K}$. That is to say, the $i$ th column of $\widehat{A}$ corresponds to $i$ th column of $\hat{\boldsymbol{\Phi}}$ and $\hat{\mathbf{S}}$. So our algorithm can estimate $2 \mathrm{D}$ DOA and frequency estimation without extra pairing.

3.2. Frequency and 2D-DOA Estimation. We use iterative least squares method to attain the direction matrix A and the delay matrix $\boldsymbol{\Phi}$, and then angle and frequency are estimated according to the least squares principle and 
Vandermonde characteristic. Define the delay vector $\mathbf{g}\left(f_{i}\right)$ as $\mathbf{g}\left(f_{i}\right)=\left[1, e^{j 2 \pi f_{i} \tau_{1}}, \ldots, e^{j 2 \pi(M-1) f_{i} \tau_{P}}\right]^{T} \cdot \mathbf{g}\left(f_{i}\right)$ is the $i$ th column of the delay matrix. We get $\mathbf{h}=\operatorname{angle}\left(\mathbf{g}\left(f_{i}\right)\right)=$ $\left[0,2 \pi f_{i} \tau_{1}, \ldots, 2 \pi f_{i}(M-1) \tau_{p}\right]^{T}$, where angle $(\cdot)$ is to get phase part of a complex number. The estimated frequency vector $\widehat{\mathbf{g}}\left(f_{i}\right)$ (the $i$ th column of the estimated delay matrix $\boldsymbol{\Phi}$ ) is processed through normalization, which also resolves the scale ambiguity, and then normalized sequence is processed to attain $\widehat{\mathbf{h}}$ according to above processing. Finally, we use leaset squares principle to estimate $f_{i}$. Least squares fitting is $\mathbf{P C}=\hat{\mathbf{h}}$, where

$$
\mathbf{P}=\left[\begin{array}{cc}
1 & 0 \\
1 & 2 \pi \tau_{1} \\
\vdots & \vdots \\
1 & 2 \pi(M-1) \tau_{P}
\end{array}\right], \quad \mathbf{C}=\left[\begin{array}{c}
c_{0} \\
f_{i}
\end{array}\right]
$$

The least squares solution for $\mathbf{C}$ is

$$
\widehat{\mathbf{C}}=\left(\mathbf{P}^{T} \mathbf{P}\right)^{-1} \mathbf{P}^{T} \hat{\mathbf{h}}
$$

Since $\mathbf{A}_{x}, \mathbf{A}_{y}$, and the delay matrix $\boldsymbol{\Phi}$ are with Vandermonde characteristic, the direction matrix $\mathbf{A}$ has a quasi-Vandermonde structure, so we can use the same method with frequency estimation method to estimate DOAs. The estimated receive array steer vector $\hat{\mathbf{a}}\left(\theta_{k}, \phi_{k}, f_{k}\right)$ (the $k$ th column of the estimated matrix $\hat{\mathbf{A}}$ ) is processed through normalization, which also resolves the scale ambiguity, then direction estimation matrix is divided into two submatrices, the first $M$ rows form submatrices $\widehat{\mathbf{A}}_{1}$. The last $M-1$ rows form sub-matrix $\hat{\mathbf{A}}_{2}$, the $k$ th column of the sub-matrix $\widehat{\mathbf{A}}_{1}$ and $\hat{\mathbf{A}}_{2}$ is $\hat{\mathbf{a}}_{1}\left(\theta_{k}, \phi_{k}, f_{k}\right)$ and $\hat{\mathbf{a}}_{2}\left(\theta_{k}, \phi_{k}, f_{k}\right)$, respectively. Then normalized sequence is processed to attain $\hat{\mathbf{g}}_{1}, \hat{\mathbf{g}}_{2}$

$$
\begin{aligned}
\hat{\mathbf{g}}_{1}= & -\operatorname{angle}\left(\hat{\mathbf{a}}_{1}\left(\theta_{k}, \phi_{k}, f_{k}\right)\right) \\
= & {\left[0, \frac{2 \pi d \cos \phi_{k} \sin \theta_{k} f_{k}}{c}, \ldots,\right.} \\
= & {\left[0, \frac{m f_{k}}{c}, \ldots, \frac{(M-1) m f_{k}}{c}\right]^{T}, } \\
\hat{\mathbf{g}}_{2}=-\operatorname{angle}\left(\hat{\mathbf{a}}_{2}\left(\theta_{k}, \phi_{k}, f_{k}\right)\right) & {\left[\frac{2 \pi d \sin \phi_{k} \sin \theta_{k} f_{k}}{c}, \ldots,\right.} \\
= & \left.\frac{2 \pi d(M-1) \sin \phi_{k} \sin \theta_{k} f_{k}}{c}\right]^{T} \sin \theta_{k} f_{k} \\
= & {\left[\frac{n f_{k}}{c}, \ldots, \frac{(M-1) n f_{k}}{c}\right]^{T}, }
\end{aligned}
$$

where $m=2 \pi d \sin \theta_{k} \cos \phi_{k}, n=2 \pi d \sin \theta_{k} \sin \phi_{k}$. Least squares fitting is $\mathbf{P}_{1} \mathbf{C}_{1}=\hat{\mathbf{g}}_{1}$, where

$$
\mathbf{P}_{1}=\left[\begin{array}{cc}
1 & 0 \\
1 & \frac{\hat{f}_{k}}{c} \\
\vdots & \vdots \\
1 & \frac{(M-1) \hat{f}_{k}}{c}
\end{array}\right], \quad \mathbf{C}_{1}=\left[\begin{array}{c}
m_{0} \\
\hat{m}
\end{array}\right],
$$

where $\hat{f}_{k}$ is the estimated frequency through (19) and $\hat{m}$ is the estimated value of $2 \pi d \sin \theta_{k} \cos \phi_{k}$. The LS solution to $m$ is

$$
\left[\begin{array}{c}
m_{0} \\
\hat{m}
\end{array}\right]=\left(\mathbf{P}_{1}^{T} \mathbf{P}_{1}\right)^{-1} \mathbf{P}_{1}^{T} \widehat{\mathbf{g}}_{1} .
$$

Similarly, $\mathbf{P}_{2} \mathbf{C}_{2}=\hat{\mathbf{g}}_{2}$, where

$$
\mathbf{P}_{2}=\left[\begin{array}{cc}
1 & \frac{\hat{f}_{k}}{c} \\
1 & \frac{2 \hat{f}_{k}}{c} \\
\vdots & \vdots \\
1 & \frac{(M-1) \hat{f}_{k}}{c}
\end{array}\right], \quad \mathbf{C}_{2}=\left[\begin{array}{c}
n_{0} \\
\hat{n}
\end{array}\right],
$$

in which $\hat{n}$ is the estimated value of $2 \pi d \sin \phi_{k} \sin \theta_{k}$. The LS solution to $n$ is

$$
\left[\begin{array}{c}
n_{0} \\
\hat{n}
\end{array}\right]=\left(\mathbf{P}_{2}^{T} \mathbf{P}_{2}\right)^{-1} \mathbf{P}_{2}^{T} \hat{\mathbf{g}}_{2} .
$$

The 2D-DOAs are estimated via

$$
\begin{gathered}
\hat{\theta}_{i}=\sin ^{-1}\left(\frac{\sqrt{\hat{m}^{2}+\hat{n}^{2}}}{2 \pi d}\right), \\
\hat{\phi}_{i}=\tan ^{-1}\left(\frac{\hat{n}}{\hat{m}}\right) .
\end{gathered}
$$

3.3. Complexity Analysis and CRB. In contrast to ESPRIT algorithms in [11] and PM algorithm in [13], our algorithm has a heavier computational load, in our method, computing $\left\{D_{p}(\boldsymbol{\Phi})\right\}_{p=1}^{P+1}$ in (14) takes a complexity of $O\left((P+1)\left[2 M K^{2}+\right.\right.$ $\left.\left.N K^{2}+K^{3}+2 M N\right]\right)$, the complexity for computing $\widehat{\widehat{S}}$ in (15) is $O\left((P+1)\left[(2 M-1) N K+N K^{2}+2 K^{2}(2 M-1)\right]+(P+\right.$ 2) $\left.\left.K^{3}+N K^{2}\right]\right)$; the complexity for computing $\hat{\mathbf{A}}$ in (17) is $O\left((P+1)\left[(2 M-1) N K+2 N K^{2}+(2 M-1) K^{2}+K^{3}\right]+K^{3}+\right.$ $\left.(2 M-1) K^{2}\right)$. Therefore, the complexity per iteration in the ILS algorithm is $O\left((P+1)\left[2(2 M-1) N K+4 N K^{2}+(8 M-\right.\right.$ 3) $\left.\left.K^{2}+3 K^{3}+2 M N\right]+2 K^{3}+(2 M+N-1) K^{2}\right)$; when the ILS algorithm converge within 20 steps, the total computational complexity of the proposed algorithm is about $O(20)(P+$ 1) $\left[2(2 M-1) N K+4 N K^{2}+(8 M-3) K^{2}+3 K^{3}+2 M N\right]+$ $\left.\left.2 K^{3}+(2 M+N-1) K^{2}\right)\right)$, and ESPRIT algorithm in [11] needs $O\left[(2 M-1)^{2}(P+1)^{2} N+(2 M-1)^{3}(P+1)^{3}+(2 M-\right.$ 1) $\left.P K^{2}+2(M-1) K^{2}+6 K^{3}\right]$, and $P M$ algorithm in [13] needs $O\left(N K[(2 M-1)(P+1)-K]+(4 M-K-3) K^{2}+4 K^{3}\right)$. 
According to [17], we derive the CRB for 2D-DOA and frequency estimation with L-shaped array

$$
\mathrm{CRB}=\frac{\sigma^{2}}{2 N}\left\{\operatorname{Re}\left[\left(\mathbf{D}^{H} \boldsymbol{\Pi}_{A}^{\perp} \mathbf{D}\right) \oplus \mathbf{P}^{T}\right]\right\}^{-1},
$$

where $\oplus$ stands for Hadamard product. $\Pi_{A}^{\perp}=\mathbf{I}-\mathbf{A}\left(\mathbf{A}^{H} \mathbf{A}\right)^{-1}$ $\mathbf{A}^{H}, \mathbf{P}=(1 / N) \sum_{n=1}^{N} \mathbf{s}\left(t_{n}\right) \mathbf{s}^{H}\left(t_{n}\right), \mathbf{D}=\left[\mathbf{d}_{1}, \mathbf{d}_{2}, \ldots, \mathbf{d}_{K}, \mathbf{f}_{1}\right.$, $\left.\mathbf{f}_{2}, \ldots, \mathbf{f}_{K}, \mathbf{g}_{1}, \mathbf{g}_{2}, \ldots, \mathbf{g}_{K}\right], \mathbf{d}_{k}=\partial \mathbf{a}_{k} / \partial \phi_{k}, \mathbf{f}_{k}=\partial \mathbf{a}_{k} / \partial \theta_{k}, \mathbf{g}_{k}=$ $\partial \mathbf{a}_{k} / \partial f_{k}$.

The advantages of the proposed algorithm can be summarized as follows. (1) The 2D-DOA and frequency can be paired automatically. (2) The proposed algorithm has better angle and frequency estimation performance than ESPRIT algorithm and PM algorithm.

\section{Simulation Results}

We present Monte Carlo simulations that are to assess joint 2D-DOA and frequency estimation performance of the proposed algorithm. The number of Monte Carlo trials is 500. There are three signals impinging on L-shaped array at $\left(15^{\circ}, 10^{\circ}, 1.2 \mathrm{MHz}\right),\left(25^{\circ}, 20^{\circ}, 1.6 \mathrm{MHz}\right),\left(35^{\circ}, 30^{\circ}\right.$, $1.8 \mathrm{MHz}$ ), respectively. We consider an L-shaped array with 15 sensors, which is shown in Figure 1. The spacing $d$ between the adjacent elements in each uniform linear array is smaller than the half smallest wavelength of the incoming signals. Define root mean squared error (RMSE) of angle:

$$
\text { RMSE }=\frac{1}{K} \sum_{k=1}^{K} \sqrt{\frac{1}{500} \sum_{m=1}^{500}\left|\alpha_{m k}-\alpha_{o k}\right|^{2}+\left|\beta_{m k}-\beta_{o k}\right|^{2}},
$$

where $\alpha_{m}$ is the estimated elevation angle, $\alpha_{o}$ is the perfect elevation angle, $\beta_{m}$ is the estimated azimuth angle, and $\beta_{o}$ is the perfect azimuth angle.

Define the root mean squared error: (RMSE) of frequency

$$
\text { RMSE }=\frac{1}{K} \sum_{k=1}^{K} \sqrt{\frac{1}{500} \sum_{m=1}^{500}\left|\gamma_{m k}-\gamma_{o k}\right|^{2}}
$$

where $r_{m}$ is the estimated frequency and $r_{o}$ is the perfect frequency. Note that $N$ is the number of snapshots; $K$ is the number of sources; $P$ is the number of delay outputs for received signal of array antennas; $L$ is the number of antennas.

Simulation 1. We first investigate the convergence performance of the proposed algorithm. Define $\delta=\left\|\widehat{\mathbf{A}}_{i}-\widehat{\mathbf{A}}_{i-1}\right\|_{F}^{2}$, where $\hat{\mathbf{A}}_{i}$ is the estimated matrix $\mathbf{A}$ of $i$ th iteration. Figure 2 presents the algorithm convergence performance of the proposed algorithm with $P=4, K=3, L=15$, and SNR = $20 \mathrm{~dB}$. Figure 2 shows that the proposed algorithm needs 20 iterations or so to achieve convergence.

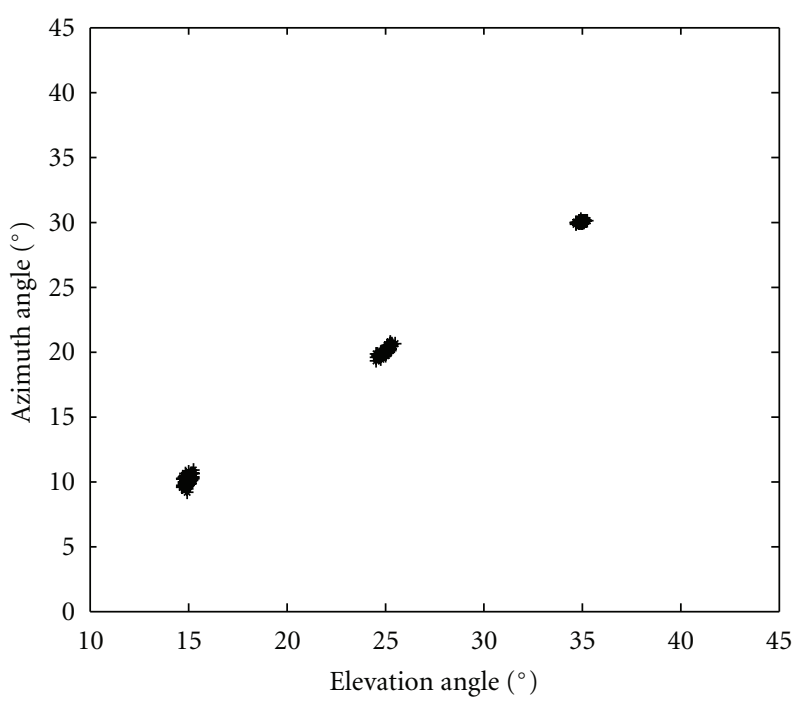

FIgURE 3: Elevation angle and azimuth angle scatter.

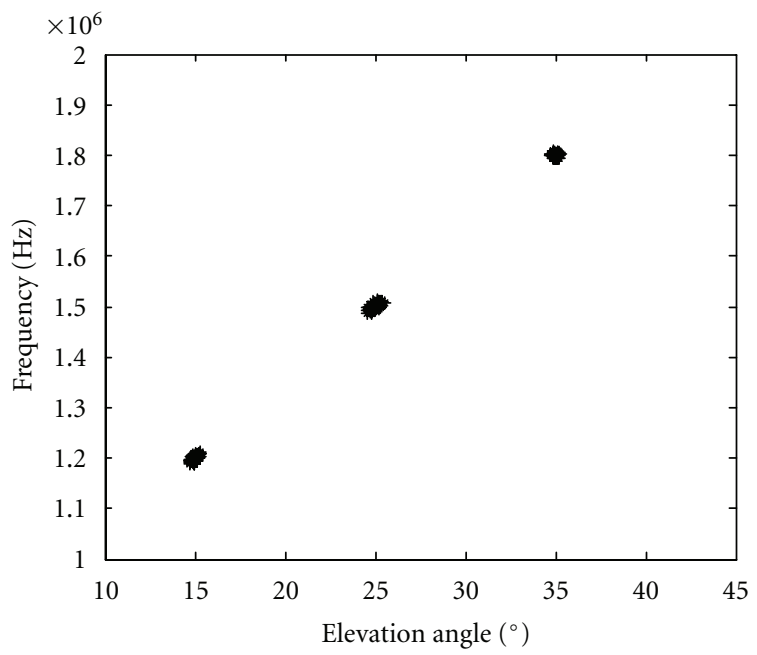

FIGURE 4: Elevation angle and frequency scatter.

Simulation 2. The performance of our proposed algorithm is investigated. $P=4, K=3, L=15$, and $N=500$ is in this simulation. Figure 3 shows $2 \mathrm{D}-\mathrm{DOA}$ estimation of our proposed algorithm at $\mathrm{SNR}=20 \mathrm{~dB}$, and Figure 4 presents elevation angle and frequency scatter of our proposed algorithm at $\mathrm{SNR}=20 \mathrm{~dB}$. From Figures 3 and 4 we find that our proposed algorithm works well.

Simulation 3. We compare our proposed algorithm with ESPRIT algorithm, MUSIC algorithm, propagator method and CRB. The simulation parameters are retained as Simulation 1. From Figures 5 and 6 we find that our algorithm has much better angle and frequency estimation performance than ESPRIT algorithm and PM algorithm, and it has a very close angle and frequency estimation performance to MUSIC algorithm, but MUSIC algorithm needs three-dimensional spectral peak searching and the complexity is much larger than our proposed algorithm. 


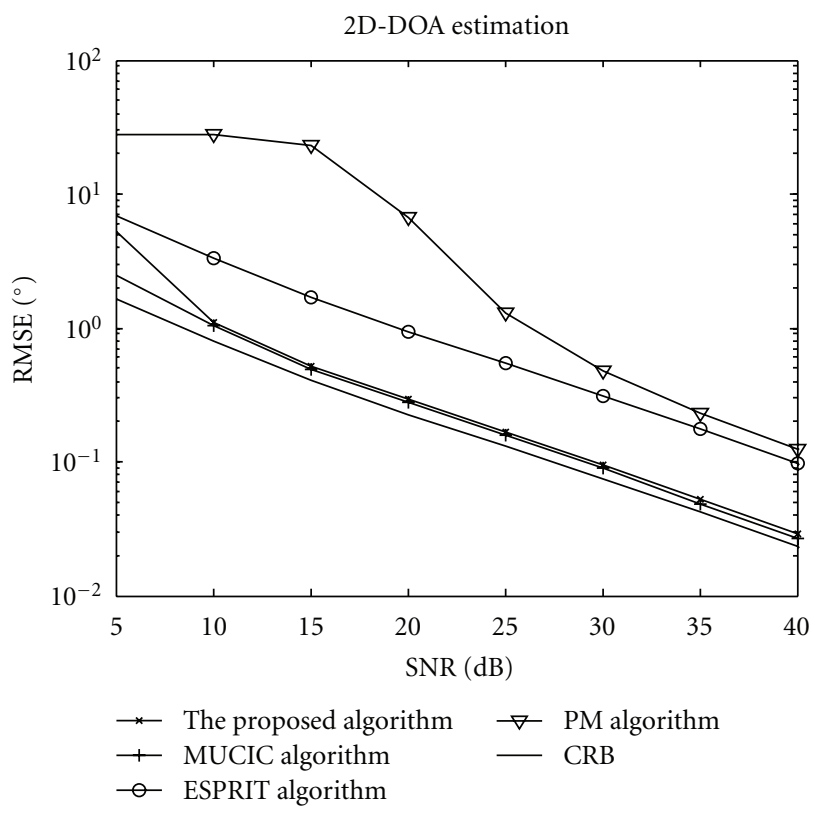

FIGURE 5: Angle estimation performance comparison.

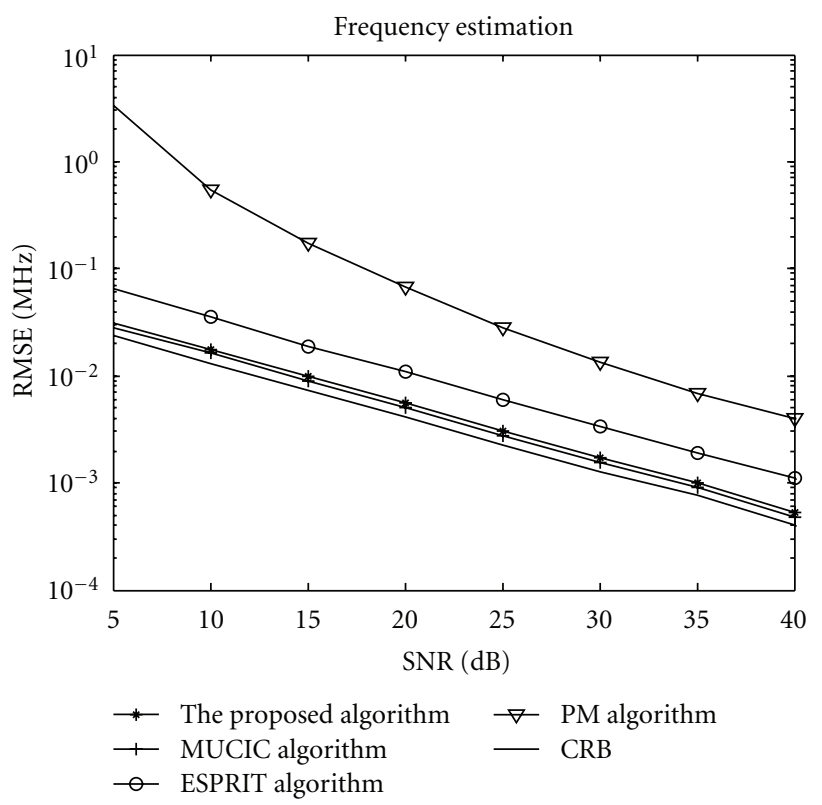

FIgURE 6: Frequency estimation performance comparison.

Simulation 4. Our proposed algorithm performance with $P=4, K=3, N=500$ and different values of $L$ is investigated. $L$ is set to 13,15 , and 17 in this simulation. It is indicated from Figures 7 and 8 that 2D-DOA estimation performance of our algorithm is improved with the number of antennas increasing. When the number of antennas increases, our algorithm has higher receive diversity.

Simulation 5. The performance of our proposed algorithm with $P=4, L=15, N=500$ and different values of $K$ is investigated. $K$ is set to $2,3,4$ in this simulation. It is

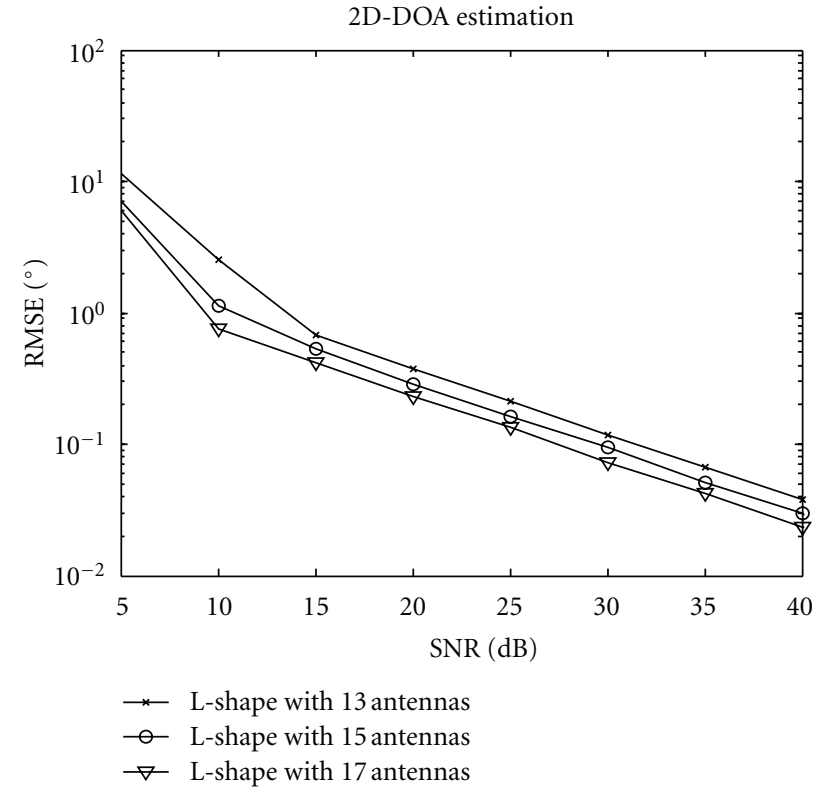

Figure 7: Angle estimation with different antennas.

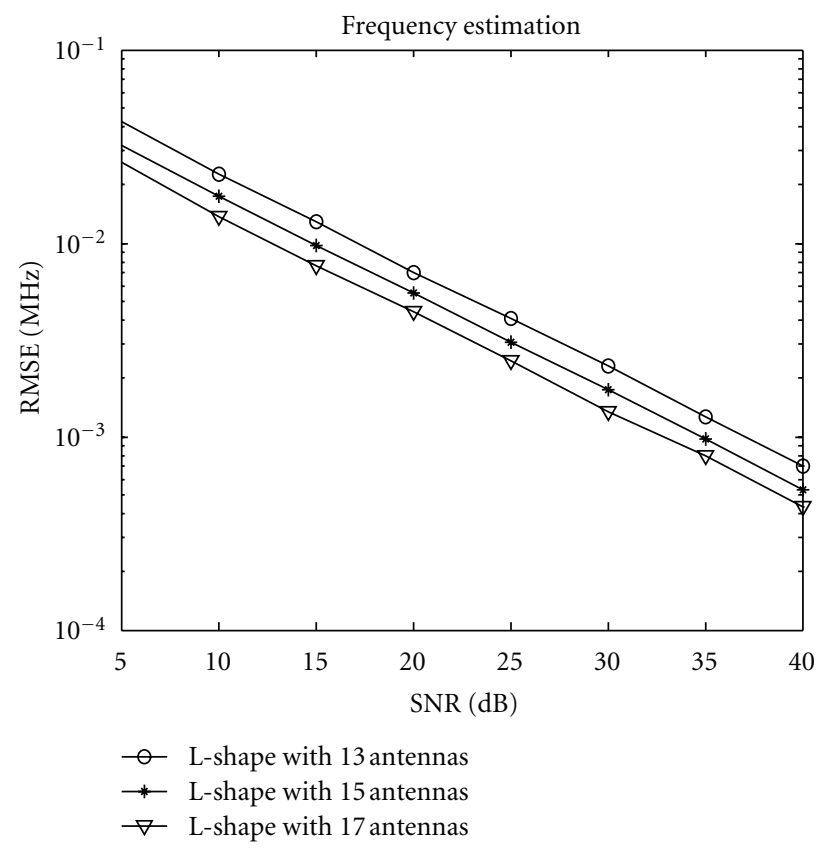

FIGURE 8: Frequency estimation with different antennas.

indicated from Figures 9 and 10 that 2D-DOA and frequency estimation performance of our algorithm degrade with the increasing of the source $K$.

\section{Conclusions}

In this paper, we develop a novel method for joint 2D-DOA and frequency estimation based on L-shaped array using iterative least squares technique. Without spectral peak 


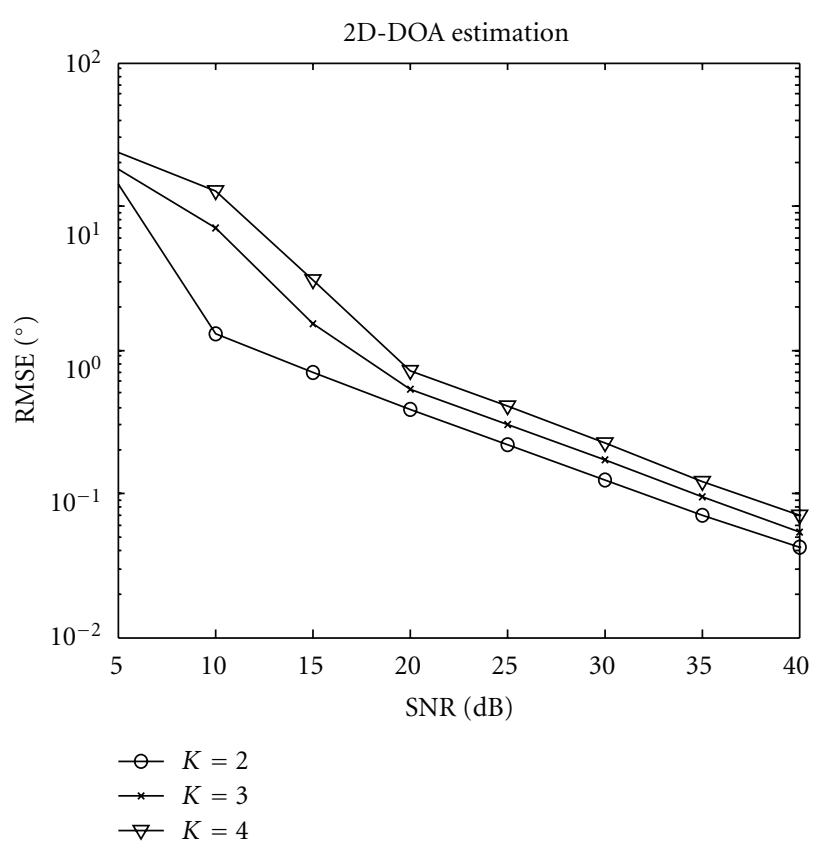

FIGURE 9: Frequency estimation with different sources.

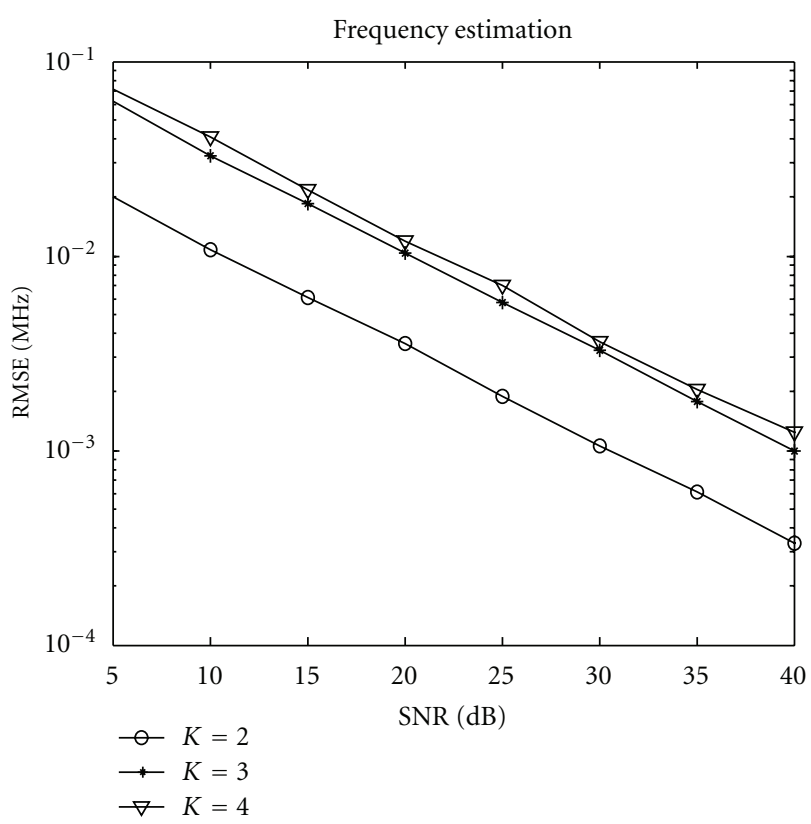

Figure 10: Frequency estimation with different sources.

searching and pairing, this algorithm works well. Furthermore, our algorithm has much better 2D-DOA and frequency estimation performance than conventional ESPRIT algorithm and PM algorithm, and it has a very close 2D-DOA and frequency estimation performance to MUSIC algorithm. The useful behavior of the proposed algorithm is verified by simulations.

\section{Acknowledgments}

This work is supported by National Nature Science Foundation of China (nos. 61179006, 60801052), Jiangsu Planned Projects for Postdoctoral Research Funds (no. 1201039C), Open project of key laboratory of underwater acoustic communication and marine information technology (Xiamen University) and Nanjing University of Aeronautics and Astronautics Research Funding (nos. NP2011036, NZ2012010, kfjj120115, kfjj20110215). The authors wish to thank the anonymous reviewers for their valuable suggestions on improving this paper.

\section{References}

[1] C. P. Mathews and M. D. Zoltowski, "Eigenstructure techniques for 2-D angle estimation with uniform circular arrays," IEEE Transactions on Signal Processing, vol. 42, no. 9, pp. 23952407, 1994.

[2] I. Bekkerman and J. Tabrikian, "Target detection and localization using MIMO radars and sonars," IEEE Transactions on Signal Processing, vol. 54, no. 10, pp. 3873-3883, 2006.

[3] F. Harabi, H. Changuel, and A. Gharsallah, "Direction of arrival estimation method using A 2-L shape arrays antenna," Progress in Electromagnetics Research, vol. 69, pp. 145-160, 2007.

[4] J. P. Lie, B. P. Ng, and C. M. S. See, "Multiple UWB emitters DoA estimation employing time hopping spread spectrum," Progress in Electromagnetics Research, vol. 78, pp. 83-101, 2008.

[5] H. X. Liu, H. Q. Zhai, L. Li, and C. H. Liang, "A progressive numerical method combined with MoM for a fast analysis of large waveguide slot antenna array," Journal of Electromagnetic Waves and Applications, vol. 20, no. 2, pp. 183-192, 2006.

[6] X. Zhang, X. Gao, and D. Xu, "Novel blind carrier frequency offset estimation for OFDM system with multiple antennas," IEEE Transactions on Wireless Communications, vol. 9, no. 3, pp. 881-885, 2010.

[7] L. Ji, J. Conan, and S. Pierre, "Joint estimation of channel parameters for MIMO communication systems," in Proceedings of the 2nd International Symposium on Wireless Communications Systems (ISWCS '05), pp. 22-26, IEEE, Siena, Italy, September 2005.

[8] M. Djeddou, A. Belouchrani, and S. Aouada, "Maximum likelihood angle-frequency estimation in partially known correlated noise for low-elevation targets," IEEE Transactions on Signal Processing, vol. 53, no. 8, pp. 3057-3064, 2005.

[9] J. D. Lin, W. H. Fang, Y. Y. Wang, and J. T. Chen, "FSF MUSIC for joint DOA and frequency estimation and its performance analysis," IEEE Transactions on Signal Processing, vol. 54, no. 12, pp. 4529-4542, 2006.

[10] Y. Y. Wang, J. T. Chen, and W. H. Fang, "TST-MUSIC for joint DOA-delay estimation," IEEE Transactions on Signal Processing, vol. 49, no. 4, pp. 721-729, 2001.

[11] A. N. Lemma, A. J. Van Der Veen, and E. F. Deprettere, "Analysis of joint angle-frequency estimation using ESPRIT," IEEE Transactions on Signal Processing, vol. 51, no. 5, pp. 12641283, 2003.

[12] A. N. Lemma, A. J. van der Veen, and E. F. Deprettere, "Joint angle-frequency estimation using multi-resolution ESPRIT," in Proceedings of the IEEE International Conference on Acoustics, Speech and Signal Processing (ICASSP '98), pp. 1957-1960, Seattle, Wash, USA, May 1998. 
[13] L. Xu, X. Zhang, and Z. Xu, "Improved joint direction of arrival and frequency estimation using propagator method," in Proceedings of the 2nd International Conference on Information Science and Engineering (ICISE '10), pp. 2139-2142, December 2010.

[14] X. Zhang, X. Gao, and W. Chen, "Improved blind 2Ddirection of arrival estimation with L-shaped array using shift invariance property," Journal of Electromagnetic Waves and Applications, vol. 23, no. 5, pp. 593-606, 2009.

[15] Z. Xiaofei, L. Jianfeng, and X. Lingyun, "Novel two-dimensional DOA estimation with L-shaped array," EURASIP Journal on Advances in Signal Processing, vol. 2011, article 50, 2011.

[16] P. Stoica and Y. Selén, "Cyclic minimizers, majorization techniques, and the expectation-maximization algorithm: a refresher," IEEE Signal Processing Magazine, vol. 21, no. 1, pp. 112-114, 2004.

[17] P. Stoica and A. Nehorai, "Performance study of conditional and unconditional direction-of-arrival estimation," IEEE Transactions on Acoustics, Speech, and Signal Processing, vol. 38, no. 10, pp. 1783-1795, 1990. 

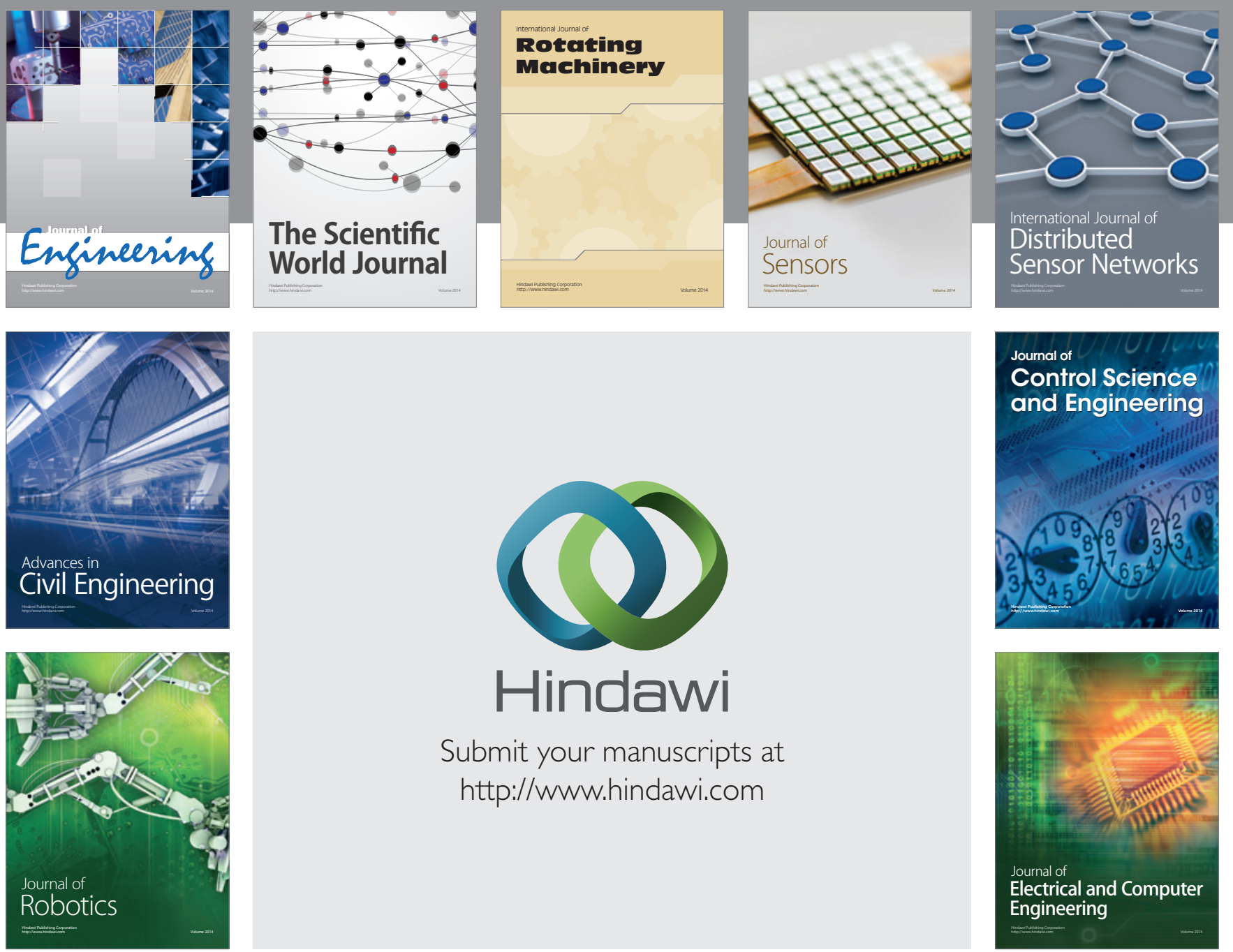

Submit your manuscripts at

http://www.hindawi.com
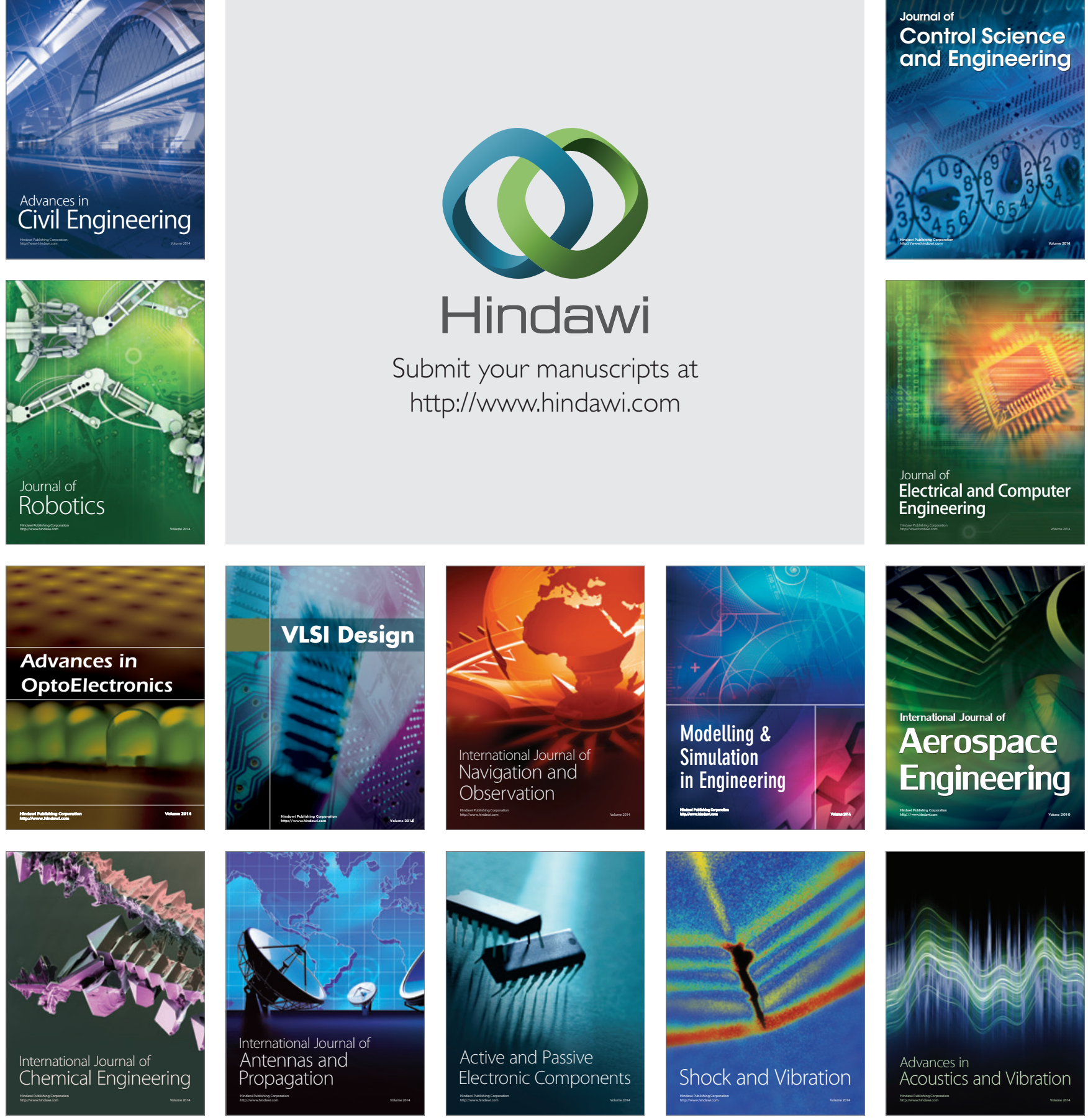